Bull. Austral. Math. Soc.

VOL. 69 (2004) [87-106]

\title{
BAER-LEVI SEMIGROUPS OF PARTIAL TRANSFORMATIONS
}

\author{
Fernanda A. Pinto and R.P. Sullivan
}

Let $X$ be an infinite set and suppose $\kappa_{0} \leqslant q \leqslant|X|$. The Baer-Levi semigroup on $X$ is the set of all injective 'total' transformations $\alpha: X \rightarrow X$ such that $|X \backslash X \alpha|=q$. It is known to be a right simple, right cancellative semigroup without idempotents, its automorphisms are "inner"; and some of its congruences are restrictions of Malcev congruences on $I(X)$, the symmetric inverse semigroup on $X$. Here we consider algebraic properties of the semigroup consisting of all injective 'partial' transformations $\alpha$ of $X$ such that $|X \backslash X \alpha|=q$ : in particular, we describe the ideals and Green's relations of it and some of its subsemigroups.

\section{INTRODUCTION}

Throughout this paper, $X$ is an infinite set with cardinal $p$, and $q$ is a cardinal such that $\aleph_{0} \leqslant q \leqslant p$. Let $P(X)$ denote the semigroup (under composition) of all partial transformations of $X$ (that is, all mappings $\alpha: A \rightarrow B$ where $A, B \subseteq X$ ). If $\alpha \in P(X)$, we write $\operatorname{dom} \alpha$ for the domain of $\alpha$ and $\operatorname{ran} \alpha$ for its range. We also write

$$
\begin{array}{ll}
G(\alpha)=X \backslash \operatorname{dom} \alpha, & g(\alpha)=|G(\alpha)| \\
D(\alpha)=X \backslash \operatorname{ran} \alpha, & d(\alpha)=|D(\alpha)|
\end{array}
$$

and refer to these cardinals as the gap and the defect of $\alpha$, respectively.

As usual, $I(X)$ denotes the symmetric inverse semigroup on $X([1$, Vol. 1, p. 29]): namely, the set of all injective mappings in $P(X)$. We write

$$
B L(q)=\{\alpha \in I(X): g(\alpha)=0, d(\alpha)=q\}
$$

and call this the Baer-Levi semigroup on $X$ : as shown in $([1$, Vol. 2, Section 8.1$])$, it is a right simple, right cancellative semigroup without idempotents; and any semigroup with these properties can be embedded in some Baer-Levi semigroup. Note that the ideals and Green's relations on $B L(q)$ are trivial. In addition, every automorphism $\varphi$

Received 7 th July, 2003

This paper forms part of work by the first author for a PhD supervised by the second author who gratefully acknowledges the generous support of Centro de Matematica, Universidade do Minho, Portugal during his visit in May-July 2002. Both authors acknowledge the support of the Portuguese Foundation for Science and Technology (FCT) through the research program POCTI.

Copyright Clearance Centre, Inc. Serial-fee code: 0004-9727/04 \$A2.00+0.00. 
of $B L(q)$ is "inner": that is, there exists $g \in G(X)$, the symmetric group on $X$, such that $\alpha \varphi=g \alpha g^{-1}$ for all $\alpha \in B L(q)$ [5]. And some congruences on $B L(q)$ are known to be restrictions of Malcev congruences on $T(X)$, the semigroup consisting of all total transformations of $X$ (that is, $\alpha \in P(X)$ such that $\operatorname{dom} \alpha=X$ ) [6].

In this paper, we examine a related semigroup:

$$
P S(q)=\{\alpha \in I(X): d(\alpha)=q\}
$$

which we call the partial Baer-Levi semigroup on $X$ (as first defined in $[9$, p. 82]). In contrast with $B L(q)$, this semigroup always contains idempotents. In fact, $P S(q)$ always contains an inverse semigroup $R(q)=\{\alpha \in P S(q): g(\alpha)=q\}$ which, together with $B L(q)$, generates $P S(q)$ in a very specific way. Also Green's relations and ideals are much more complicated. In Sections 4 and 5 we describe the latter for both $P S(q)$ and $R(q)$ : this will be the basis for subsequent work regarding the congruences on $P S(q)$.

\section{BASIC PROPERTIES}

In what follows, $Y=A \dot{\cup} B$ means $Y$ is a disjoint union of $A$ and $B$. Also, $\emptyset$ denotes the empty (one-to-one) mapping which acts as a zero for $P(X)$. In particular, $d(\emptyset)=p$, so $\emptyset \in P S(q)$ precisely when $q=p$. For each non-empty $A \subseteq X$, we write $\mathrm{id}_{A}$ for the identity transformation on $A$ : these mappings constitute all the idempotents in $I(X)$ and belong to $P S(q)$ precisely when $|X \backslash A|=q$.

We adopt the convention introduced in [1, Vol. 2, p. 241]: namely, if $\alpha \in P(X)$ is non-zero then we write

$$
\alpha=\left(\begin{array}{c}
A_{i} \\
x_{i}
\end{array}\right)
$$

and take as understood that the subscript $i$ belongs to some (unmentioned) index set $I$, that the abbreviation $\left\{x_{i}\right\}$ denotes $\left\{x_{i}: i \in I\right\}$, and that $X \alpha=\operatorname{ran} \alpha=\left\{x_{i}\right\}, x_{i} \alpha^{-1}$ $=A_{i}$ and $\operatorname{dom} \alpha=\bigcup\left\{A_{i}: i \in I\right\}$.

Recall that a semigroup $S$ is right reductive if $a x=b x$ for all $x \in S$ implies $a=b$ (and dually for left reductive: see [1, Vol. 1, p. 9]).

THEOREM 1. If $\aleph_{0} \leqslant q \leqslant p$ then $P S(q)$ is a right and left reductive semigroup with idempotents. Moreover, $P S(q)$ contains a zero precisely when $q=p$.

Proof: If $\alpha, \beta \in P S(q)$, we have

$$
\begin{aligned}
X \backslash X \alpha \beta & =X \backslash X \beta \cup[X \beta \backslash X \alpha \beta] \\
& =X \backslash X \beta \cup[(X \backslash X \alpha) \cap \operatorname{dom} \beta] \beta
\end{aligned}
$$


and in the last equation, the first set on the right has cardinal $q$ and the second has cardinal at most $q$, thus $\alpha \beta \in P S(q)$. Also $P S(q)$ contains idempotents since we can write $X=A \cup \dot{\cup} B$ where $|A|=p,|B|=q$ and then $\operatorname{id}_{A} \in P S(q)$. In addition, if $\zeta$ is a zero for $P S(q)$ then $\zeta=\zeta$. id $A$, hence $\operatorname{ran} \zeta \subseteq A$, for all $A \subseteq X$ such that $|X \backslash A|=q$. In particular, if $x \notin D(\zeta)$ and we choose $B \subseteq X$ such that $x \notin B$ and $|X \backslash(B \cup\{x\})|=q$ then $D(\zeta)$ contains $B \cup\{x\}$, a contradiction. Thus, every element of $X$ belongs to $D(\zeta)$ and this occurs only when $q=p$.

To show $P S(q)$ is right reductive, suppose $\alpha, \beta \in P S(q)$ and $\alpha \gamma=\beta \gamma$ for all $\gamma \in P S(q)$. If $\alpha, \beta \neq \emptyset$ then $\operatorname{id}_{X \alpha} \in P S(q)$, so $\alpha=\alpha$. id $X_{\alpha}=\beta$. id $\operatorname{id}_{\alpha}$ and this implies $X \alpha \subseteq X \beta$. The reverse inclusion also holds since $\operatorname{id}_{X \beta} \in P S(q)$. Hence $X \alpha=X \beta$ and it follows that $\alpha=\beta$. If (say) $\alpha=\emptyset$ then $q=p$ and $\beta \gamma=\emptyset$ for all $\gamma \in P S(q)$. In particular, $\beta$. id $\{b\}=\emptyset$ for all $b \in X \beta$ and thus $\beta=\emptyset$.

Now suppose $\gamma \alpha=\gamma \beta$ for all $\gamma \in P S(q)$. If $\alpha, \beta \neq \emptyset$, let $b \in \operatorname{dom} \alpha$ and write $X=\{b\} \dot{\cup}\left\{x_{i}\right\} \dot{\cup}\left\{x_{j}\right\}$ where $|I|=p,|J|=q$. Then

$$
\gamma=\left(\begin{array}{ll}
x_{i} & b \\
x_{i} & b
\end{array}\right) \in P S(q)
$$

and $b \in \operatorname{dom} \gamma \alpha=\operatorname{dom} \gamma \beta$, so $b \in \operatorname{dom} \beta$. Hence, $\operatorname{dom} \alpha \subseteq \operatorname{dom} \beta$ and the reverse inclusion also holds. It follows that $b \alpha=b \beta$ for all $b \in \operatorname{dom} \alpha=\operatorname{dom} \beta$ and hence $\alpha=\beta$. If (say) $\alpha=\emptyset$ and $x \in X$ then, as before, $\operatorname{id}_{\{x\}} \in P S(q)$, so $\operatorname{id}_{\{x\}} . \beta=\emptyset$ for all $x \in X$ and this implies $\beta=\emptyset$.

EXAMPLE 1. Unlike $B L(q)$, the semigroup $P S(q)$ is not right cancellative nor right simple. For, suppose $X=A \cup \dot{\cup} B$ where $|A|=p,|B|=q, A=\left\{a_{i}\right\}$ and $b, c \in B$ are distinct. If

$$
\alpha=\left(\begin{array}{ll}
a_{i} & b \\
a_{i} & b
\end{array}\right), \beta=\left(\begin{array}{ll}
a_{i} & b \\
a_{i} & c
\end{array}\right)
$$

then $\alpha, \beta \in P S(q)$ and $\alpha \cdot \operatorname{id}_{A}=\beta \cdot \operatorname{id}_{A}$ but $\alpha \neq \beta$. Also, suppose $X=A \dot{\cup} B \dot{\cup} C$ where $|A|=p$ and $|B|=|C|=q$. If $\alpha=\operatorname{id}_{A \cup B}$ and $\beta=\operatorname{id}_{A \cup C}$, both of which are in $P S(q)$, then $C \cap \operatorname{dom} \alpha \gamma=\emptyset$ for each $\gamma \in P S(q)$. Therefore, since $C \subset \operatorname{dom} \beta$, there is no $\gamma \in P S(q)$ such that $\beta=\alpha \gamma$ : that is, $P S(q)$ is not right simple.

A subsemigroup $S$ of $P(X)$ is $G(X)$-normal if $g \alpha g^{-1} \in S$ for all $\alpha \in S$ and all $g \in G(X)$. Clearly $P S(q)$ is $G(X)$-normal and, if $q=p$, then $P S(q)$ covers $X$ : that is, for each $x \in X$, there is an idempotent constant map (namely, $\left.\operatorname{id}_{\{x\}}\right)$ in $P S(q)$ with range $\{x\}$. Hence, by [9] Theorem 3, if $q=p$ then every automorphism of $P S(q)$ is 'inner' (as defined in Section 1 above) and moreover Aut $P S(q)$ is isomorphic to $G(X)$. When $q<p, P S(q)$ does not contain any constant maps. Nonetheless, by $[4$, Theorem 3.18], every automorphism of $P S(q)$ is inner in this case also. 
We aim to show that Aut $P S(q)$ is also isomorphic to $G(X)$ when $q<p$. For this, we first need to know that if $\varphi \in \operatorname{Aut} P S(q)$ then there exists a unique $h \in G(X)$ such that $\alpha \varphi=h^{-1} \alpha h$ for all $\alpha \in P S(q)$. In other words, if $h, k \in G(X)$ and $h^{-1} \alpha h=k^{-1} \alpha k$ for all $\alpha \in P S(q)$ then $h=k$. To show this, we use some ideas from [5] and let

$$
\mathcal{C}(p, q)=\{A \subseteq X:|A|=p,|X \backslash A|=q\}
$$

If $A \in C(p, q)$ and $\alpha$ is any bijection from $X$ onto $A$ then $\alpha \in P S(q)$ and $X h^{-1} \alpha h$ $=A h$, so $A h=A k$ for all $A \in C(p, q)$. Fix $x \in X$ and write $X=A \dot{\cup} B \dot{\cup}\{x\}$ where $|A|=p$ and $|B|=q$. Since $h$ and $k$ are injective,

$$
(A \cup\{x\}) h=A h \cup \dot{\cup}\{x\} \text { and }(A \cup\{x\}) k=A h \dot{\cup}\{x\} k .
$$

Therefore, since $(A \cup\{x\}) h=(A \cup\{x\}) k$, we find that $x h=x k$ for all $x \in X$, hence $h=k$. We can now prove the following result.

Theorem 2. If $q<p$ then Aut $P S(q)$ is isomorphic to $G(X)$.

Proof: Let $\theta:$ Aut $P S(q) \rightarrow G(X), \varphi \rightarrow h_{\varphi}$, where $h_{\varphi}$ is the unique permutation of $X$ such that $\alpha \varphi=h_{\varphi}^{-1} \alpha h_{\varphi}$ for all $\alpha \in P S(q)$. To show $\theta$ is a morphism, let $\varphi, \psi \in$ Aut $P S(q)$ and note that for all $\alpha \in P S(q)$, we have:

$$
\alpha(\varphi \psi)=\left(h_{\varphi} h_{\psi}\right)^{-1} \alpha\left(h_{\varphi} h_{\psi}\right)
$$

hence $h_{\varphi \psi}=h_{\varphi} h_{\psi}$ by uniqueness. Clearly, if $k \in G(X)$ then

$$
\varphi: P S(q) \rightarrow P S(q), \alpha \rightarrow k^{-1} \alpha k
$$

is an automorphism of $P S(q)$ (since $P S(q)$ is $G(X)$-normal). Thus, $h_{\varphi}=k$ by uniqueness, so $\theta$ is onto. Finally, if $h_{\varphi}=h_{\psi}$ then $\alpha \varphi=\alpha \psi$ for all $\alpha \in P S(q)$, so $\varphi=\psi$ and $\theta$ is one-to-one.

In what follows, we sometimes write $P S(X, p, q)$ or $P S(p, q)$ in place of $P S(q)$ to highlight the underlying set $X$ or its cardinal $p$.

As might be expected, $P S(X, p, q)$ is isomorphic to $P S(Y, r, s)$ if and only if $p=r$ and $q=s$, and moreover each isomorphism is induced in a natural way by a bijection from $X$ onto $Y$. To prove this, we need an argument almost identical to that in [5]. However, since we are dealing with partial transformations and our argument differs in some important respects, we provide all the details.

LEMMA 1. If $\alpha, \beta \in P S(p, q)$ then the following are equivalent.

(a) $\operatorname{ran} \alpha \subseteq \operatorname{ran} \beta$,

(b) for each $\gamma \in P S(p, q), \beta \gamma=\beta$ implies $\alpha \gamma=\alpha$. 
Proof: If $\operatorname{ran} \alpha \subseteq \operatorname{ran} \beta$ and $\beta \gamma=\beta$ for some $\gamma \in P S(q)$ then $(x \alpha) \gamma=x \alpha$ for each $x \alpha \in \operatorname{ran} \beta$, so $\alpha \gamma=\alpha$. Conversely, suppose there exists $y=x \alpha \notin \operatorname{ran} \beta=B$ say. Then $\operatorname{id}_{B} \in P S(q)$ and $\beta \circ \mathrm{id}_{B}=\beta$ but $y \operatorname{id}_{B} \neq y$; that is, $\alpha \circ \mathrm{id}_{B} \neq \alpha$ and hence the condition does not hold.

Suppose $|X|=p \geqslant q \geqslant \aleph_{0}$ and let $\mathcal{B}(X, q)$ denote the family of all $A \subseteq X$ such that $|X \backslash A|=q$. Note that the poset $(\mathcal{B}(X, q), \subseteq)$ contains a least element if and only if $p=q$, and in this case $\emptyset$ is its least element. For, clearly if $p=q$ then $\emptyset \in \mathcal{B}(X, q)$. And if $q<p$ then each $A \in \mathcal{B}(X, q)$ is non-empty and $A \backslash\{x\} \in \mathcal{B}(X, q)$; that is, $\mathcal{B}(X, q)$ cannot contain a least element in this case. The proof of the next result closely follows the corresponding argument in [5].

Lemma 2. Suppose $|X|=p \geqslant q \geqslant \aleph_{0}$ and $|Y|=r \geqslant s \geqslant \aleph_{0}$. Every orderisomorphism $H: \mathcal{B}(X, q) \rightarrow \mathcal{B}(Y, s)$ is induced by a bijection $h: X \rightarrow Y:$ that is, for each $A \in \mathcal{B}(X, q)$, we have $A H=A h$, the image of $A$ under $h$.

Proof: Let $A \in \mathcal{B}(X, q)$ and $x \in X \backslash A$. We write $A \cup\{x\}$ as $A \cup x$. Clearly, $A \cup x \in \mathcal{B}(X, q)$ and $A \cup x$ covers $A$. Hence $(A \cup x) H=A H \cup y$ for some $y \notin A H$. We write $y=x h_{A}$ and assert that $x h_{A}=x h_{B}$ for all $A, B \in \mathcal{B}(X, q)$ not containing $x$. For, clearly $A \cap B \in \mathcal{B}(X, q)$ and, since $H$ is an order-isomorphism, $(A \cap B) H=A H \cap B H$. Therefore, as in the proof of [5, Lemma, p. 493],

$$
\begin{aligned}
(A H \cap B H) \cup x h_{A \cap B} & =(A \cap B) H \cup x h_{A \cap B} \\
& =((A \cap B) \cup x) H \\
& =((A \cup x) \cap(B \cup x)) H \\
& =(A \cup x) H \cap(B \cup x) H \\
& =\left(A H \cup x h_{A}\right) \cap\left(B H \cup x h_{B}\right),
\end{aligned}
$$

and it follows that

$$
\left\{x h_{A \cap B}\right\}=\left(A H \cap\left\{x h_{B}\right\}\right) \cup\left(\left\{x h_{A}\right\} \cap B H\right) \cup\left(\left\{x h_{A}\right\} \cap\left\{x h_{B}\right\}\right) .
$$

Now if $x h_{B} \in A H$ then $x h_{A \cap B}=x h_{B}$ and hence

$$
((A \cap B) \cup x) H=(A \cap B) H \cup x h_{A \cap B}=(A \cap B) H \cup x h_{B} \subseteq A H .
$$

This implies $(A \cap B) \cup x \subseteq A$, contradicting $x \notin A$. Therefore, $x h_{B} \notin A H$ and similarly $x h_{A} \notin B H$. Hence $\left\{x h_{A}\right\} \cap\left\{x h_{B}\right\} \neq \emptyset$ and this means $x h_{A}=x h_{B}$ as asserted.

Now define $h: X \rightarrow Y, x \mapsto x h_{A}$, where $A \in \mathcal{B}(X, q)$ satisfies $x \notin A$. The above argument shows $h$ is well-defined. To see $h$ is injective, suppose $x_{1} h=x_{2} h$ and choose 
$B \in \mathcal{B}(X, q)$ such that $x_{i} \notin B$ for $i=1,2$. Then, by definition, $x_{i} h=x_{i} h_{B}$ for $i=1,2$. Therefore

$$
\left(B \cup x_{1}\right) H=B H \cup x_{1} h_{B}=B H \cup x_{2} h_{B}=\left(B \cup x_{2}\right) H
$$

and it follows that $x_{1}=x_{2}$. To show $h$ is surjective, let $y \in Y$ and choose $M \in \mathcal{B}(Y, s)$ such that $y \notin M$. Then $A H=M$ and $B H=M \cup y$ for some $A, B \in \mathcal{B}(X, q)$. Since $M \cup y$ covers $M$ in the poset $\mathcal{B}(Y, s), B$ must cover $A$ in the poset $\mathcal{B}(X, q)$. That is, $B=A \cup x$ for some $x \notin A$. Hence

$$
M \cup y=(A \cup x) H=A H \cup x h_{A}=M \cup x h_{A}
$$

and it follows that $y=x h_{A}$ and thus $y=x h$ by definition. That is, $h$ is a bijection.

Finally we prove that $A H=A h$ for each $A \in \mathcal{B}(X, q)$. First recall that the empty map $\emptyset \in P S(q)$ if and only if $p=q$. In this case, the empty set $\emptyset$ is the least element of $\mathcal{B}(X, q)$ and hence $\emptyset H$ is a least element for $\mathcal{B}(Y, s)$. This means $r=s$ and $\emptyset H=\emptyset=\emptyset h$. So we can assume $A \in \mathcal{B}(X, q)$ is non-empty. Now if $y=x h$ for some $x \in A$ then $y=x h_{B}$ where $x \notin B \in \mathcal{B}(X, q)$. If $y \notin A H$ then $A H \cup y \in \mathcal{B}(Y, s)$ and $A H \cup y=(A \cup z) H$ for some $z \notin A$. Hence $z h_{A}=y=x h_{B}$ and, since $h$ is injective, this implies $z=x \in A$, a contradiction. Therefore $y \in A H$ and $A h \subseteq A H$. Conversely, if $y \in A H$ then $A H$ covers $A H \backslash y$ (this is true even if $A H=\{y\}$, which is possible when $p=q)$. Hence $A H \backslash y=(A \backslash x) H$ for some $x \in A$ and so

$$
A H=((A \backslash x) \cup x) H=(A \backslash x) H \cup x h_{A \backslash x}
$$

Therefore, since $y \notin(A \backslash x) H$, we know $y=x h_{A \backslash x}$ and this means $y=x h \in A h$; that is, $A H \subseteq A h$ and equality follows.

Recall that $P S(p, q)$ contains a zero element (namely, $\emptyset$ ) precisely when $p=q$. Consequently, if $P S(X, p, q)$ and $P S(Y, r, s)$ are isomorphic then either $p=q$ and $r=s$, or $p>q$ and $r>s$. In what follows, we need the fact: if $A, B \subseteq X$ and $\alpha \in I(X)$ then $(A \backslash B) \alpha=A \alpha \backslash B \alpha$.

THEOREM 3. The semigroups $P S(X, p, q)$ and $P S(Y, r, s)$ are isomorphic if and only if $p=r$ and $q=s$. Moreover, for each isomorphism $\varphi$, there is a bijection $h: X \rightarrow Y$ such that $\alpha \varphi=h^{-1} \alpha h$ for each $\alpha \in P S(X, p, q)$.

Proof: Clearly, if the cardinals are equal as stated, then any bijection from $X$ onto $Y$ will induce an isomorphism between the semigroups. So we assume there is an isomorphism $\varphi: P S(X, p, q) \rightarrow P S(Y, r, s)$ and aim to find a bijection $h: X \rightarrow Y$. First we observe that $\varphi$ induces an order-isomorphism from $\mathcal{B}(X, q)$ onto $\mathcal{B}(Y, s)$. Indeed, from Lemma 1 we deduce that, for each $\alpha, \beta \in P S(q), \operatorname{ran} \alpha=\operatorname{ran} \beta$ if and 
only if $\operatorname{ran}(\alpha \varphi)=\operatorname{ran}(\beta \varphi)$. Also, recall that $\operatorname{id}_{A} \in P S(q)$ for each $A \in \mathcal{B}(X, q)$. Consequently, there is a well-defined mapping

$$
H: \mathcal{B}(X, q) \rightarrow \mathcal{B}(Y, s), A \mapsto \operatorname{ran}(\alpha \varphi)
$$

where $A=\operatorname{ran} \alpha$ for some $\alpha \in P S(q)$. Note that if $p=q$ and $A=\emptyset=\operatorname{ran} \emptyset$ where $\emptyset \in P S(q)$ then $\emptyset \varphi=\emptyset$ and $\emptyset H=\emptyset$. More generally, if $A, B \in \mathcal{B}(X, q)$ and $A=\operatorname{ran} \alpha, B=\operatorname{ran} \beta$ for some $\alpha, \beta \in P S(q)$ then $A H=\operatorname{ran}(\alpha \varphi), B H=\operatorname{ran}(\beta \varphi)$, and $A \subseteq B$ if and only if $A H \subseteq B H$ by Lemma 1. Also, for each $M \in \mathcal{B}(Y, s)$, there exists $\gamma \in P S(s)$ and $\alpha \in P S(q)$ such that $M=\operatorname{ran} \gamma$ and $\gamma=\alpha \varphi$ : that is, $M=(\operatorname{ran} \alpha) H$ where $\operatorname{ran} \alpha \in \mathcal{B}(X, q)$, hence $H$ is surjective.

By Lemma 2, $H$ is induced by a bijection $h: X \rightarrow Y$ and now we aim to show $\alpha \varphi=h^{-1} \alpha h$ for each $\alpha \in P S(q)$. Clearly this holds if $p=q$ and $\alpha=\emptyset$. So, suppose $\alpha \neq \emptyset$ and note that $\operatorname{dom} \alpha h=\operatorname{dom} \alpha \operatorname{since} \operatorname{dom} h=X$. Let $x \in \operatorname{dom} \alpha$ and $x \alpha=x^{\prime}$. Choose $A, B$ in $\mathcal{B}(X, q)$ such that $A \subseteq B$ and $B \backslash A=\{x\}$, and consider $\beta, \gamma \in P S(X, q)$ such that $\operatorname{ran} \beta=A$ and $\operatorname{ran} \gamma=B$. Now $\operatorname{ran} \gamma \backslash \operatorname{ran} \beta=\{x\}$ and so

$$
\begin{aligned}
\operatorname{ran}((\gamma \alpha) \varphi) \backslash \operatorname{ran}((\beta \alpha) \varphi) & =\operatorname{ran}((\gamma \varphi)(\alpha \varphi)) \backslash \operatorname{ran}((\beta \varphi)(\alpha \varphi)) \\
& =(\operatorname{ran}(\gamma \varphi) \backslash \operatorname{ran}(\beta \varphi))(\alpha \varphi) \\
& =(B H \backslash A H)(\alpha \varphi) \\
& =\{x h\} \alpha \varphi
\end{aligned}
$$

On the other hand, $\operatorname{ran}(\gamma \alpha) \backslash \operatorname{ran}(\beta \alpha)=(B \backslash A) \alpha=\left\{x^{\prime}\right\}$ and so

$$
\begin{aligned}
\operatorname{ran}((\gamma \alpha) \varphi) \backslash \operatorname{ran}((\beta \alpha) \varphi) & =(\operatorname{ran}(\gamma \alpha)) H \backslash(\operatorname{ran}(\beta \alpha)) H \\
& =(\operatorname{ran}(\gamma \alpha)) h \backslash(\operatorname{ran}(\beta \alpha)) h \\
& =(\operatorname{ran} \gamma \backslash \operatorname{ran} \beta) \alpha h \\
& =\left\{x^{\prime} h\right\}
\end{aligned}
$$

Thus $x h(\alpha \varphi)=x^{\prime} h=x \alpha h$ for all $x \in \operatorname{dom} \alpha$ and so $\alpha \varphi=h^{-1} \alpha h$. Finally, since $\alpha \varphi \in P S(Y, r, s)$ implies $|Y \backslash Y \alpha \varphi|=s$, whereas $\left|Y \backslash Y h^{-1} \alpha h\right|=|(X \backslash X \alpha) h|=q$ for any bijection $h: X \rightarrow Y$, we also have $q=s$.

\section{Regular elements}

Since $B L(q)$ is idempotent-free, it contains no regular elements (if $S$ is a semigroup, we say $a \in S$ is regular if $a=a x a$ for some $x \in S$ ). But $P S(q)$ always contains regular elements. 
THEOREM 4. If $\aleph_{0} \leqslant q \leqslant p$ and $\alpha \in P S(q)$ then the following statements are equivalent.
(a) $\alpha$ is regular,
(b) $g(\alpha)=q$,
(c) $\alpha^{-1} \in P S(q)$.

Proof: Suppose $\alpha=\alpha \beta \alpha$ for some $\beta \in P S(q)$. Then, since $\alpha$ is injective, $x \alpha \beta=x$ for all $x \in \operatorname{dom} \alpha$ and hence $\operatorname{dom} \alpha \subseteq \operatorname{ran} \beta$. Therefore, $q=d(\beta)$ $\leqslant g(\alpha)$. Suppose $g(\alpha)=r>q$. Then $X \backslash \operatorname{dom} \alpha=(\operatorname{ran} \beta \backslash \operatorname{dom} \alpha) \cup(X \backslash X \beta)$ implies $|\operatorname{ran} \beta \backslash \operatorname{dom} \alpha|=r$. That is, if $\operatorname{ran} \beta \backslash \operatorname{dom} \alpha=\left\{d_{k}\right\}$ where $|K|=r$ and $c_{k} \beta=d_{k}$ then $\left\{c_{k}\right\} \cap \operatorname{ran} \alpha=\emptyset$ (since $\alpha \beta=\operatorname{id}_{\operatorname{dom} \alpha}$ ) and so $\left\{c_{k}\right\} \subseteq X \backslash \operatorname{ran} \alpha$ which implies $d(\alpha)$ $\geqslant r>q$, a contradiction. This proves (a) implies (b). If $g(\alpha)=q$ then $d\left(\alpha^{-1}\right)=q$, so $\alpha^{-1} \in P S(q)$; and if $\alpha^{-1} \in P S(q)$ then clearly $\alpha$ is a regular element of $P S(q)$.

The set of regular elements in $P S(q)$ plays an important role in what follows, so we let

$$
R(q)=\{\alpha \in P S(q): g(\alpha)=q\} .
$$

Clearly any regular subsemigroup of $P S(q)$ is contained in $R(q)$. Therefore, the next result shows that $R(q)$ is the largest regular subsemigroup of $P S(q)$. In fact, since all idempotents of $P S(q)$ have the form id ${ }_{A}$ for some $A \subseteq X$ and all of these commute, we see that every regular subsemigroup of $P S(q)$ is inverse.

COROLlary 1. If $\aleph_{0} \leqslant q \leqslant p$ then $R(q)$ is an inverse semigroup.

Proof: The idempotents in $P S(q)$ commute and, by the above Theorem, $R(q)$ is regular, so it remains to show $R(q)$ is closed. Suppose $\alpha, \beta \in R(q)$ and note that

$$
\operatorname{dom} \alpha \beta=(\operatorname{ran} \alpha \cap \operatorname{dom} \beta) \alpha^{-1} \subseteq X \alpha^{-1},
$$

so

$$
\begin{aligned}
X \backslash \operatorname{dom} \alpha \beta & =X \backslash X \alpha^{-1} \cup\left[X \alpha^{-1} \backslash(\operatorname{ran} \alpha \cap \operatorname{dom} \beta) \alpha^{-1}\right] \\
& =X \backslash X \alpha^{-1} \cup[X \backslash(\operatorname{ran} \alpha \cap \operatorname{dom} \beta)] \alpha^{-1}
\end{aligned}
$$

where the first set on the right of (1) has cardinal $q$ (since $\alpha^{-1} \in P S(q)$ by the Theorem). Also, $X \backslash[\operatorname{ran} \alpha \cap \operatorname{dom} \beta]=(X \backslash \operatorname{ran} \alpha) \cup(X \backslash \operatorname{dom} \beta)$, so the second set on the right of (1) has cardinal at most $q$ (since $\alpha^{-1}$ is injective). Therefore, $g(\alpha \beta)=q$, and we have shown $\alpha \beta \in R(q)$.

REMARK 1. In [3], Howie used $R(q)=\{\alpha \in I(X): d(\alpha)=g(\alpha)=q\}$ to construct a congruence-free inverse semigroup when $p>q$; and in [10, Corollary 4], Sullivan 
showed that $R(p)$ is generated by its nilpotents with index 2: in fact, it equals the subsemigroup of $I(X)$ generated by all the nilpotents in $I(X)$.

For $\aleph_{0} \leqslant r \leqslant p$, we write

$$
S_{r}=\{\alpha \in P S(q): g(\alpha) \leqslant r\}
$$

This is a subsemigroup of $P S(q)$ since if $\alpha, \beta \in S_{r}$ then

$$
g(\alpha \beta)=\left|X \backslash X \alpha^{-1}\right| \cup\left|[X \backslash(\operatorname{ran} \alpha \cap \operatorname{dom} \beta)] \alpha^{-1}\right|
$$

where $X \backslash X \alpha^{-1}=X \backslash$ dom $\alpha$, regardless of whether $\alpha^{-1} \in P S(q)$. Hence, $g(\alpha \beta)$ $\leqslant r+(0+r)=r$, so $\alpha \beta \in S_{r}$. In particular,

$$
B L(q) \cup R(q) \subset S_{q}
$$

and so the two semigroups on the left cannot generate $S_{r}$ for any $r>q$. In addition, if $\gamma \in P S(q)$ and $\gamma=\alpha \beta$ for some $\alpha \in R(q)$ and $\beta \in B L(q)$ then $g(\gamma) \geqslant g(\alpha)$. Hence $R(q) . B L(q)$ is a proper subset of $S_{q}$. On the other hand, the next two results show that $S_{q}$ is generated by $B L(q)$ and $R(q)$ in very specific ways: this will be important when we consider maximal subsemigroups of $P S(q)$ in a subsequent paper.

THEOREM 5. If $\aleph_{0} \leqslant q \leqslant p$ then $S_{q}=B L(q) \cdot R(q)$. In fact, $S_{q}=\alpha \cdot R(q)$ for each $\alpha \in B L(q)$.

Proof: We have already seen that $B L(q) \cdot R(q) \subseteq S_{q}$. For the converse, suppose $\alpha \in S_{q}$ and note that

$$
X \backslash X \alpha=[(X \backslash X \alpha) \cap \operatorname{dom} \alpha] \cup[(X \backslash X \alpha) \cap(X \backslash \operatorname{dom} \alpha)]
$$

Hence, if $g(\alpha)<q$ then the second intersection on the right has cardinal less than $q$, whereas the set on the left of the equation has cardinal equal to $q$, hence we have:

$$
|(X \backslash X \alpha) \cap \operatorname{dom} \alpha|=q
$$

Write $(X \backslash X \alpha) \cap \operatorname{dom} \alpha=\left\{a_{i}\right\}=\left\{b_{i}\right\} \dot{\cup}\left\{c_{i}\right\} \dot{\cup}\left\{d_{j}\right\}$ where $|J|=g(\alpha)<q$, and let $\operatorname{dom} \alpha \cap \operatorname{ran} \alpha=\left\{x_{k}\right\}$ and $X \backslash \operatorname{dom} \alpha=\left\{y_{j}\right\}$. Let

$$
\lambda=\left(\begin{array}{ccc}
x_{k} & a_{i} & y_{j} \\
x_{k} & b_{i} & d_{j}
\end{array}\right), \mu=\left(\begin{array}{cc}
x_{k} & b_{i} \\
x_{k} \alpha & a_{i} \alpha
\end{array}\right)
$$

which are well-defined one-to-one maps by construction. Moreover, $\operatorname{dom} \lambda=X$ and $X \backslash X \lambda=\left\{c_{i}\right\} \cup\left\{y_{j}\right\}$ : that is, $\lambda \in B L(q)$; and $X \backslash \operatorname{dom} \mu=\left\{c_{i}\right\} \cup\left\{d_{j}\right\} \cup\left\{y_{j}\right\}$ and $X \backslash X \mu=X \backslash X \alpha$ : that is, $\mu \in R_{q}$. And clearly $\alpha=\lambda \mu$. 
If $g(\alpha)=q$, we can write $\operatorname{dom} \alpha=\left\{u_{k}\right\}, X \backslash \operatorname{dom} \alpha=\left\{y_{j}\right\}$ and $X \backslash X \alpha$ $=\left\{v_{j}\right\} \dot{\cup}\left\{w_{j}\right\}$ where $|J|=q$. Let

$$
\lambda=\left(\begin{array}{cc}
u_{k} & y_{j} \\
u_{k} \alpha & v_{j}
\end{array}\right), \mu=\mathrm{id}_{X \alpha} \in R_{q}
$$

Then $\lambda$ is a well-defined element of $B L(q)$ and $\lambda \mu=\alpha$ as required.

Finally, suppose $\alpha, \beta \in B L(q)$, let $X=\left\{x_{i}\right\}$ and write

$$
\alpha=\left(\begin{array}{c}
x_{i} \\
a_{i}
\end{array}\right), \quad \beta=\left(\begin{array}{c}
x_{i} \\
b_{i}
\end{array}\right), \quad \mu=\left(\begin{array}{c}
a_{i} \\
b_{i}
\end{array}\right) .
$$

Then $\beta=\alpha \mu$ where $\mu \in R(q)$, so $B L(q) \subseteq \alpha . R(q) \subseteq S_{q}$. On the other hand, if $\gamma \in S_{q}$ then the above argument shows $\gamma=\beta \mu$ for some $\beta \in B L(q)$ and some $\mu \in R(q)$, and now we also know $\beta=\alpha \lambda$ for some $\lambda \in R(q)$. Therefore, $\gamma=\alpha(\lambda \mu)$ where $\lambda \mu \in R(q)$ since $R(q)$ is a semigroup; that is, $S_{q} \subseteq \alpha . R(q)$ and equality follows.

The next result shows that in most cases $S_{q}$ can be generated in a different way.

THEOREM 6. If $q<p$ then $S_{q}=B L(q) . \mu \cdot B L(q)$ for each $\mu \in R(q)$.

Proof: Suppose $\gamma \in S_{q}$ with $g(\gamma)=r$ and let $\mu \in R(q)$. Since $q<p$, both $\gamma$ and $\mu$ have rank $p$, so we can write

$$
\gamma=\left(\begin{array}{c}
a_{i} \\
b_{i}
\end{array}\right), \quad \mu=\left(\begin{array}{c}
c_{i} \\
d_{i}
\end{array}\right)
$$

Let $X \backslash\left\{a_{i}\right\}=\left\{a_{j}\right\}$ (so $|J|=r$ ), $X \backslash\left\{c_{i}\right\}=\left\{y_{j}\right\} \cup\left\{y_{k}\right\}$ where $|K|=q, X \backslash\left\{d_{i}\right\}=\left\{d_{k}\right\}$ and $X \backslash\left\{b_{i}\right\}=\left\{u_{k}\right\} \dot{\cup}\left\{v_{k}\right\}$. If

$$
\alpha=\left(\begin{array}{ll}
a_{i} & a_{j} \\
c_{i} & y_{j}
\end{array}\right), \quad \beta=\left(\begin{array}{ll}
d_{i} & d_{k} \\
b_{i} & u_{k}
\end{array}\right)
$$

then $\alpha, \beta \in B L(q)$ and $\gamma=\alpha \mu \beta$ (note that if $r=0$ then $\left\{a_{j}\right\}=\emptyset$ but the conclusion is the same).

In passing we note that if $\gamma \in S_{q}, \mu \in R(q)$ and $\gamma=\alpha \mu \beta$ for some $\alpha, \beta \in B L(q)$ then $\operatorname{dom} \gamma \subseteq \operatorname{dom} \alpha$, so $(\operatorname{dom} \gamma) \alpha \subseteq \operatorname{dom} \mu$ and hence $|\operatorname{dom} \gamma| \leqslant|\operatorname{dom} \mu|=r(\mu)$. Therefore, if $q=p$ and $r(\mu)<p$ then $g(\gamma)=g(\mu)=p$, so $B L(q)$. $\mu . B L(q)$ is a proper subset of $S_{q}$; that is, the above result fails to hold when $q=p$. In addition, it cannot be simplified to read, for example: $S_{q}=\mu . B L(q)$ for each $\mu \in R(q)$ when $q<p$. For, if $\gamma \in S_{q}$ then $\gamma \neq \mu \beta$ for each $\mu \in R(q)$ such that $\operatorname{dom} \gamma \mathbb{E} \operatorname{dom} \mu$. A similar argument using ran $\gamma$ shows that also $S_{q} \neq B L(q) . \mu$ for some $\mu \in R(q)$. 


\section{GREEN'S RELATions}

The semigroup $P S(q)$ is not a regular subsemigroup of $P(X)$, so Hall's Theorem ([2, Proposition II.4.5]) cannot be used to describe the $\mathcal{L}$ and $\mathcal{R}$ relations on $P S(q)$ in terms of their well-known characterisation on $P(X)$ (see [7, Theorem 10]). Therefore, in this section we first characterise each of the Green's relations on $P S(q)$ and then consider the corresponding problem for $S_{q}$ and $R(q)$. In fact, for each of these semigroups, $S$ say, we determine when $S^{1} \alpha \subseteq S^{1} \beta$ and $\alpha S^{1} \subseteq \beta S^{1}$ for $\alpha, \beta \in S$ (that is, when $\mathcal{L}$ and $\mathcal{R}$ classes are comparable under their usual partial order).

THEOREM 7. If $\alpha, \beta \in P S(q)$ then $\alpha=\beta \mu$ for some $\mu \in P S(q)$ if and only if $\operatorname{dom} \alpha \subseteq \operatorname{dom} \beta$. Hence $\alpha \mathcal{R} \beta$ in PS(q) if and only if $\operatorname{dom} \alpha=\operatorname{dom} \beta$.

Proof: Clearly, if $\alpha=\beta \mu$ for some $\mu \in P S(q)$ then $\operatorname{dom} \alpha \subseteq \operatorname{dom} \beta$. Conversely, suppose $\operatorname{dom} \alpha \subseteq \operatorname{dom} \beta$ and write

$$
\alpha=\left(\begin{array}{c}
a_{i} \\
b_{i}
\end{array}\right), \quad \beta=\left(\begin{array}{cc}
a_{i} & x_{j} \\
c_{i} & y_{j}
\end{array}\right), \quad \mu=\left(\begin{array}{c}
c_{i} \\
b_{i}
\end{array}\right) .
$$

Then $\alpha=\beta \mu$ where $\mu \in P S(q)$.

Surprisingly, it is much harder to describe Green's $\mathcal{L}$ relation on $P S(q)$.

THEOREM 8. If $\alpha, \beta \in P S(q)$ then $\alpha=\lambda \beta$ for some $\lambda \in P S(q)$ if and only if $X \alpha \subseteq X \beta$ and

$$
q \leqslant \max (g(\beta),|X \beta \backslash X \alpha|) \leqslant \max (g(\alpha), q) .
$$

Hence, $\alpha \mathcal{L} \beta$ in $P S(q)$ if and only if

$$
(X \alpha=X \beta \text { and } g(\alpha)=g(\beta) \geqslant q) \text { or }(\alpha=\beta \text { and } g(\alpha)<q) \text {. }
$$

Proof: Suppose $\alpha=\lambda \beta$ for some $\lambda \in P S(q)$. Then $X \alpha \subseteq X \beta$ and $\alpha \in P S(q)$ implies

$$
|[(X \backslash X \lambda) \cap \operatorname{dom} \beta] \beta|=|(X \backslash X \lambda) \beta|=|X \beta \backslash X \alpha| \leqslant d(\alpha)=q .
$$

Also, since $\beta$ is one-to-one, we have:

$$
\begin{aligned}
q=|X \backslash X \lambda| & =|[(X \backslash X \lambda) \cap \operatorname{dom} \beta] \cup[(X \backslash X \lambda) \cap(X \backslash \operatorname{dom} \beta)]| \\
& \leqslant|X \beta \backslash X \alpha|+g(\beta)=\max (g(\beta),|X \beta \backslash X \alpha|) .
\end{aligned}
$$

Since $\lambda$ is one-to-one and $\alpha=\lambda \beta$, we have

$$
(X \lambda \cap \operatorname{dom} \beta) \lambda^{-1}=\operatorname{dom} \alpha \text { and }(X \lambda \cap X \backslash \operatorname{dom} \beta) \lambda^{-1} \subseteq X \backslash \operatorname{dom} \alpha
$$


and hence

$$
\begin{aligned}
|X \backslash \operatorname{dom} \beta| & =|X \lambda \cap(X \backslash \operatorname{dom} \beta)|+|(X \backslash X \lambda) \cap(X \backslash \operatorname{dom} \beta)| \\
& \leqslant|X \backslash \operatorname{dom} \alpha|+q=\max (g(\alpha), q) .
\end{aligned}
$$

Conversely, suppose $\alpha, \beta \in P S(q)$ and the conditions hold. Write

$$
\alpha=\left(\begin{array}{c}
a_{i} \\
b_{i}
\end{array}\right), \beta=\left(\begin{array}{ll}
x_{i} & x_{k} \\
b_{i} & b_{k}
\end{array}\right), \lambda=\left(\begin{array}{l}
a_{i} \\
x_{i}
\end{array}\right)
$$

so that $|K|=|X \beta \backslash X \alpha|$. If $g(\alpha)<q$, the conditions imply $\max (g(\beta),|X \beta \backslash X \alpha|)=q$ and so $d(\lambda)=\left|\left\{x_{k}\right\} \cup(X \backslash \operatorname{dom} \beta)\right|=q$; that is, $\lambda \in P S(q)$. Suppose $g(\alpha) \geqslant q$. In this case, the conditions imply $g(\beta) \leqslant g(\alpha)$ : otherwise, we have

$$
|X \beta \backslash X \alpha| \leqslant q \leqslant g(\alpha)<g(\beta)
$$

and so

$$
\max (g(\beta),|X \beta \backslash X \alpha|)=g(\beta)>g(\alpha)=\max (g(\alpha), q)
$$

We can also assume $q<g(\beta)$ : otherwise, $\max (g(\beta),|X \beta \backslash X \alpha|)=q$ and the result follows as before. Now write $X \backslash \operatorname{dom} \beta=\left\{x_{m}\right\} \dot{\cup}\left\{x_{n}\right\}$ where $|M|=g(\beta),|N|=q$ and choose $z_{m} \in X \backslash$ dom $\alpha$. Now re-define $\lambda$ as

$$
\lambda=\left(\begin{array}{ll}
a_{i} & z_{m} \\
x_{i} & x_{m}
\end{array}\right)
$$

and note that $X \backslash X \lambda=\left\{x_{k}\right\} \cup\left\{x_{n}\right\}$ which has cardinal $q$. Hence, $\lambda \in P S(q)$ and $\alpha=\lambda \beta$ as required.

It follows that for distinct $\alpha, \beta \in P S(q), \alpha=\lambda \beta$ and $\beta=\lambda^{\prime} \alpha$ for some $\lambda, \lambda^{\prime}$ $\in P S(q)$ if and only if $X \alpha=X \beta$ and $g(\alpha)=g(\beta) \geqslant q$. That is, if $\alpha \mathcal{L} \beta$ in $P S(q)$ and $g(\alpha) \geqslant q$ then $X \alpha=X \beta$ and $g(\alpha)=g(\beta)$, whereas if $g(\alpha)<q$ then $\alpha=\beta$. On the other hand, if one of these events occurs, it is now clear that $\alpha \mathcal{L} \beta$ in $P S(q)$.

Given that the condition in (2) is so complicated, it is worth noting that it cannot be simplified to read: $g(\beta) \leqslant g(\alpha)$.

EXAMPLE 2. Let $\alpha, \beta \in P S(q)$ be defined by

$$
\alpha=\left(\begin{array}{c}
a_{i} \\
b_{i}
\end{array}\right), \beta=\left(\begin{array}{ll}
x_{i} & x_{j} \\
b_{i} & b_{j}
\end{array}\right)
$$

where $g(\beta) \leqslant g(\alpha)<q$ and $|J|<q$. Note that in this case $X \alpha \subseteq X \beta$ and $|I|=p$. Also $\max (g(\beta),|X \beta \backslash X \alpha|) \ngtr q$. If $\alpha=\lambda \beta$ for some $\lambda \in P S(q)$ then $b_{i}=a_{i} \alpha$ $=\left(a_{i} \lambda\right) \beta=x_{i} \beta$ for each $i$, so $\left\{x_{i}\right\} \subseteq X \lambda$. Therefore

$$
d(\lambda) \leqslant\left|X \backslash\left\{x_{i}\right\}\right|=\left|\left\{x_{j}\right\} \cup G(\beta)\right|<q+q=q,
$$

a contradiction. That is, for some $\alpha, \beta \in P S(q)$ with $g(\beta) \leqslant g(\alpha)$, there is no $\lambda$ $\in P S(q)$ such that $\alpha=\lambda \beta$. 
REMARK 2. From Theorems 7 and 8 , we deduce that $\alpha \mathcal{H} \beta$ in $P S(q)$ if and only if

$$
(X \alpha=X \beta, \operatorname{dom} \alpha=\operatorname{dom} \beta \text { and } g(\alpha) \geqslant q) \text { or }(\alpha=\beta \text { and } g(\alpha)<q) \text {. }
$$

Recall that each group $\mathcal{H}$-class of $T(X)$ is isomorphic to a symmetric group $G(A)$ for some $A \subseteq X$ ([1, Vol. 1, Theorem 2.10]). The corresponding result for $P S(q)$ is even more precise. For, if $\varepsilon$ is a non-zero idempotent of $P S(q)$ then $\varepsilon=\mathrm{id}_{A}$ for some $A \subseteq X$ such that $|X \backslash A|=q$. Consequently, since each $\alpha \in P . S(q)$ is injective, we have

$$
\begin{aligned}
\alpha \in H_{\varepsilon} & \Longleftrightarrow X \alpha=X \varepsilon, \operatorname{dom} \alpha=\operatorname{dom} \varepsilon, \\
& \Longleftrightarrow \operatorname{ran} \alpha=\operatorname{dom} \alpha=A, \\
& \Longleftrightarrow \alpha \in G(A) .
\end{aligned}
$$

That is, $H_{\varepsilon}=G(A)$ and clearly, when $p=q, H_{\emptyset}=\{\emptyset\}$.

To characterise the $\mathcal{J}$ relation on $P S(q)$, we need two Lemmas. Henceforth, if $\alpha \in P(X)$, we write $r(\alpha)=|\operatorname{ran} \alpha|$ and call this the rank of $\alpha$.

Lemma 3. If $q<p$ and $\alpha, \beta \in P S(q)$ then $\beta=\lambda \alpha \mu$ for some $\lambda, \mu \in P S(q)$ if and only if $g(\alpha) \leqslant q$ or $g(\beta) \geqslant g(\alpha)>q$. Hence, in $P S(q)$ for $q<p, \alpha \mathcal{J} \beta$ if and only if $g(\alpha)$ and $g(\beta)$ are at most $q$, or $g(\alpha)=g(\beta)>q$.

PRoOF: First note that if $q<p$ then $r(\alpha)=r(\beta)=p$. Suppose $\beta=\lambda \alpha \mu$ for some $\lambda, \mu \in P S(q)$ and assume $g(\alpha)=r>q$. Then

$$
|(X \backslash X \lambda) \cap(X \backslash \operatorname{dom} \alpha)| \leqslant q<r
$$

and this implies $|X \lambda \cap G(\alpha)|=r$. That is, there exists $\left\{a_{n}\right\} \subseteq \operatorname{dom} \lambda$ such that $|N|=r$ and $\left\{a_{n} \lambda\right\} \cap \operatorname{dom} \alpha=\emptyset$. Therefore, $\left\{a_{n}\right\} \subseteq G(\beta)$ and $g(\beta) \geqslant r=g(\alpha)$, as required. Conversely, if $g(\alpha) \leqslant q<p$, write

$$
\beta=\left(\begin{array}{c}
c_{i} \\
d_{i}
\end{array}\right) \text { and } \alpha=\left(\begin{array}{c}
a_{i} \\
b_{i}
\end{array}\right)
$$

where $|I|=p$ and let $\left\{a_{i}\right\}=\left\{x_{i}\right\} \dot{\cup}\left\{x_{j}\right\}$ where $|J|=q$. Define

$$
\lambda=\left(\begin{array}{c}
c_{i} \\
x_{i}
\end{array}\right) \text { and } \mu=\left(\begin{array}{c}
x_{i} \alpha \\
d_{i}
\end{array}\right)
$$

and note that $D(\lambda)=\left\{x_{j}\right\} \cup G(\alpha)$, a set with cardinal $q$. Moreover, $\beta=\lambda \alpha \mu$ where $\lambda, \mu \in P S(q)$. On the other hand, if $g(\beta) \geqslant g(\alpha)=r>q$, choose $n_{j} \in G(\alpha)$ with $|J|=r$ and $\left|G(\alpha) \backslash\left\{n_{j}\right\}\right|=q$, and choose $m_{j} \in G(\beta)$ (possible via the assumption). Then, using the same notation for $\alpha$ and $\beta$, we see that

$$
\lambda=\left(\begin{array}{cc}
c_{i} & m_{j} \\
a_{i} & n_{j}
\end{array}\right) \text { and } \mu=\left(\begin{array}{c}
b_{i} \\
d_{i}
\end{array}\right)
$$


are elements of $P S(q)$ and $\beta=\lambda \alpha \mu$, as required.

LEMMA 4. If $q=p$ and $\alpha, \beta \in P S(q)$ then $\beta=\lambda \alpha \mu$ for some $\lambda, \mu \in P S(q)$ if and only if $r(\beta) \leqslant r(\alpha)$. Hence, in $P S(q)$ for $q=p, \alpha \mathcal{J} \beta$ if and only if $r(\alpha)=r(\beta)$.

Proof: Clearly, $\beta=\lambda \alpha \mu$ implies $r(\beta) \leqslant r(\alpha)$. For the converse, write

$$
\beta=\left(\begin{array}{c}
c_{j} \\
d_{j}
\end{array}\right) \text { and } \alpha=\left(\begin{array}{c}
a_{i} \\
b_{i}
\end{array}\right)
$$

Put $\left\{a_{i}\right\}=\left\{x_{j}\right\} \dot{\cup}\left\{x_{k}\right\}$ (possible since $r(\beta) \leqslant r(\alpha)$ ) and define

$$
\lambda=\left(\begin{array}{c}
c_{j} \\
x_{j}
\end{array}\right) \text { and } \mu=\left(\begin{array}{c}
x_{j} \alpha \\
d_{j}
\end{array}\right)
$$

and note that $D(\lambda)=\left\{x_{k}\right\} \cup G(\alpha)$ : clearly, this set has cardinal $q=p$ if $g(\alpha)=q$; and if $g(\alpha)<q$ then $|I|=q$, so we can ensure that $|K|=q$. That is, $\lambda, \mu \in P S(q)$ and $\beta=\lambda \alpha \mu$.

Note that $g(\alpha)>q$ can occur only when $q<p$; and if $g(\alpha) \leqslant q<p$ then $r(\alpha)=p$. Also, if $q=p$ then $\max (g(\alpha), g(\beta)) \leqslant q$ is valid for all $\alpha, \beta \in P S(q)$. Hence the last two Lemmas can be combined as follows.

THEOREM 9. If $\aleph_{0} \leqslant q \leqslant p$ then $\alpha \mathcal{J} \beta$ in $P S(q)$ if and only if

$$
[\max (g(\alpha), g(\beta)) \leqslant q \text { and } r(\alpha)=r(\beta)] \text { or }[g(\alpha)=g(\beta)>q] .
$$

We now consider the $\mathcal{D}$ relation on $P S(q)$ and find that $\mathcal{D} \neq \mathcal{J}$, unlike the usual situation for other subsemigroups of $P(X)$ (for example, the semigroup generated by the idempotents of $T(X)$ [8, Theorem 7], and the semigroup generated by the nilpotents of $P(X)[7$, Theorem 11]).

THEOREM 10. If $\aleph_{0} \leqslant q \leqslant p$ then $\alpha \mathcal{D} \beta$ in PS(q) if and only if

$$
[g(\alpha)<q \text { and } \operatorname{dom} \alpha=\operatorname{dom} \beta] \text { or }[r(\alpha)=r(\beta) \text { and } g(\alpha)=g(\beta) \geqslant q] \text {. }
$$

Proof: Suppose $\alpha \mathcal{L} \gamma \mathcal{R} \beta$ in $P S(q)$. By Theorems 8 and 7, " $\alpha=\gamma$ and $g(\alpha)<q$ " or " $X \alpha=X \gamma$ and $g(\gamma)=g(\alpha) \geqslant q$ ", and $\operatorname{dom} \gamma=\operatorname{dom} \beta$. Since $\gamma$ and $\beta$ are one-to-one on their domains, we deduce that

$$
[g(\alpha)<q \text { and } \operatorname{dom} \alpha=\operatorname{dom} \beta] \text { or }[r(\alpha)=r(\beta) \text { and } g(\alpha)=g(\beta) \geqslant q] .
$$

Conversely, suppose this condition holds. If $g(\alpha)<q$ and $\operatorname{dom} \alpha=\operatorname{dom} \beta$, then $\alpha \mathcal{L} \alpha \mathcal{R} \beta$. On the other hand, if $r(\alpha)=r(\beta)$ and $g(\alpha)=g(\beta) \geqslant q$, we write

$$
\alpha=\left(\begin{array}{c}
a_{i} \\
b_{i}
\end{array}\right), \quad \beta=\left(\begin{array}{c}
c_{i} \\
d_{i}
\end{array}\right), \quad \gamma=\left(\begin{array}{c}
c_{i} \\
b_{i}
\end{array}\right) .
$$

Then $\gamma \in P S(q)$ and, by Theorems 8 and $7, \alpha \mathcal{L} \gamma \mathcal{R} \beta$ as required. 
Example 3. Let $\alpha, \beta \in P S(q)$ be defined by

$$
\alpha=\left(\begin{array}{c}
a_{i} \\
b_{i}
\end{array}\right), \beta=\left(\begin{array}{c}
c_{i} \\
d_{i}
\end{array}\right)
$$

where $g(\beta)<g(\alpha)<q$ and $\operatorname{dom} \alpha \neq \operatorname{dom} \beta$. This implies $|I|=p$, so $r(\alpha)=r(\beta)$ and $\max (g(\alpha), g(\beta))<q$, hence $\alpha \mathcal{J} \beta$ by Theorem 9. Suppose $\alpha \mathcal{L} \gamma \mathcal{R} \beta$ for some $\gamma \in P S(q)$. Then $\operatorname{dom} \gamma=\operatorname{dom} \beta$ by Theorem 7, hence $\alpha \neq \gamma$ (by choice). So Theorem 8 implies $X \alpha=X \gamma$ and $g(\alpha)=g(\gamma) \geqslant q$, contradicting the choice of $\alpha$. Hence $\alpha$ is not $\mathcal{D}$-related to $\beta$ in $P S(q)$, and thus $\mathcal{D} \neq \mathcal{J}$.

We now consider Green's relations on $S_{q}$. As before, since $S_{q}$ is not a regular subsemigroup of $P S(q)$, Hall's Theorem cannot be applied to find $\mathcal{R}$ and $\mathcal{L}$ on $S_{q}$. Nonetheless, they happen to be the restriction of $\mathcal{R}$ and $\mathcal{L}$ on $P S(q)$.

Lemma 5. Let $\alpha, \beta \in S_{q}$ where $\aleph_{0} \leqslant q \leqslant p$. Then

(a) $\alpha=\beta \mu$ for some $\mu \in S_{q}$ if and only if $\operatorname{dom} \alpha \subseteq \operatorname{dom} \beta$, and

(b) $\alpha=\lambda \beta$ for some $\lambda \in S_{q}$ if and only if $X \alpha \subseteq X \beta$ and $\max (g(\beta),|X \beta \backslash X \alpha|)$ $=q$.

Proof: For (a), we simply note that in the proof of Theorem 7, if $\alpha \in S_{q}$ then $\left\{x_{j}\right\} \subseteq G(\alpha)$, so $|J| \leqslant q$ and $G(\mu)=\left\{y_{j}\right\} \cup D(\beta)$, hence $g(\mu) \leqslant q$.

For (b), observe that if $\alpha=\lambda \beta$ for some $\lambda \in S_{q} \subseteq P S(q)$ then the condition in Theorem 8 simplifies to the desired result. Conversely, suppose the stated condition holds and write

$$
\alpha=\left(\begin{array}{c}
a_{i} \\
b_{i}
\end{array}\right), \beta=\left(\begin{array}{ll}
x_{i} & x_{j} \\
b_{i} & b_{j}
\end{array}\right), \lambda=\left(\begin{array}{c}
a_{i} \\
x_{i}
\end{array}\right) .
$$

Then $|J| \leqslant q$ since $|X \beta \backslash X \alpha| \leqslant d(\alpha)=q$. If $g(\beta)=q$ then $d(\lambda)=g(\beta)+|J|=q$ and clearly $g(\lambda) \leqslant q$, so $\lambda \in S_{q}$ and $\alpha=\lambda \beta$. On the other hand, if $|X \beta \backslash X \alpha|=q$ then $|J|=q \geqslant g(\beta)$ and again $d(\lambda)=q$, so $\lambda \in S_{q}$ as required.

CoRollary 2. Let $\alpha, \beta \in S_{q}$ where $\aleph_{0} \leqslant q \leqslant p$. Then

(a) $\alpha \mathcal{R} \beta$ in $S_{q}$ if and only if $\operatorname{dom} \alpha=\operatorname{dom} \beta$, and

(b) $\alpha \mathcal{L} \beta$ in $S_{q}$ if and only if $[X \alpha=X \beta$ and $g(\alpha)=g(\beta)=q]$ or $[\alpha=\beta$ and $g(\alpha)<q]$.

From Lemma 3 we see that if $q<p$ then $S_{q}$ forms a $\mathcal{J}$-class in $P S(q)$. Hence we might expect the $\mathcal{J}$ relation on $S_{q}$ to be universal when $q<p$. In addition, given the last result, we might also expect the $\mathcal{D}$ relation on $S_{q}$ to be the restriction of $\mathcal{D}$ on $P S(q)$. Both these expectations are correct, as we now show.

THEOREM 11. Let $\alpha, \beta \in S_{q}$ where $\aleph_{0} \leqslant q \leqslant p$. Then $\beta=\lambda \alpha \mu$ for some $\lambda, \mu$ $\in S_{q}$ if and only if $r(\beta) \leqslant r(\alpha)$. Hence

(a) $\quad \alpha \mathcal{J} \beta$ in $S_{q}$ if and only if $r(\alpha)=r(\beta)$, and 
(b) $\alpha \mathcal{D} \beta$ in $S_{q}$ if and only if $[g(\alpha)<q$ and $\operatorname{dom} \alpha=\operatorname{dom} \beta]$ or $[r(\alpha)$ $=r(\beta)$ and $g(\alpha)=g(\beta)=q]$.

Proof: Clearly, $\beta=\lambda \alpha \mu$ implies $r(\beta) \leqslant r(\alpha)$. Conversely, if $q<p$ then $r(\alpha)$ $=r(\beta)=p$. Using the same notation as in the proof of Lemma 3, we note that $g(\lambda)$ $=g(\beta) \leqslant q$ and $G(\mu)=D(\alpha) \cup\left\{x_{j} \alpha\right\}$, a set with cardinal $q$, so $\lambda, \mu \in S_{q}$ in this case. On the other hand, if $q=p$ and $r(\beta) \leqslant r(\alpha)$ then we observe that the $\lambda, \mu$ defined in the proof of Lemma 4 actually belong to $S_{q}$.

It remains to prove (b). If $\alpha \mathcal{D} \beta$ in $S_{q}$ then $\alpha \mathcal{D} \beta$ in $P S(q)$, so Theorem 10 gives the desired result. Conversely, if the condition holds, we note that the converse argument in the proof of Theorem 10 shows in fact that $\gamma \in S_{q}$ and hence $\alpha \mathcal{D} \beta$ in $S_{q}$.

We now turn to Green's relations on $R(q)$. Since this is a regular subsemigroup of $I(X)$, Hall's Theorem implies that the $\mathcal{L}$ and $\mathcal{R}$ relations on $R(q)$ equal the restriction of the corresponding relations on $I(X)$ to $R(q)$. Hence, $\alpha \mathcal{L} \beta$ in $R(q)$ if and only if $\operatorname{ran} \alpha=\operatorname{ran} \beta$, and $\alpha \mathcal{R} \beta$ in $R(q)$ if and only if $\operatorname{dom} \alpha=\operatorname{dom} \beta$. In fact, the $\mathcal{J}$ and $\mathcal{D}$ relations on $R(q)$ also mimic those on $I(X)$.

THEOREM 12. If $\alpha, \beta \in R(q)$ then $\beta=\lambda \alpha \mu$ for some $\lambda, \mu \in R(q)$ if and only if $r(\beta) \leqslant r(\alpha)$. Hence, $\alpha \mathcal{J} \beta$ in $R(q)$ if and only if $r(\alpha)=r(\beta)$. Consequently, $\mathcal{D}=\mathcal{J}$ in $R(q)$.

Proof: As usual, if $\beta=\lambda \alpha \mu$ for some $\lambda, \mu \in P(X)$ then $r(\beta) \leqslant r(\alpha)$. Conversely, if this condition holds, we write

$$
\alpha=\left(\begin{array}{c}
a_{i} \\
b_{i}
\end{array}\right), \quad \beta=\left(\begin{array}{c}
c_{j} \\
d_{j}
\end{array}\right), \quad \lambda=\left(\begin{array}{c}
c_{j} \\
x_{j}
\end{array}\right), \quad \mu=\left(\begin{array}{c}
x_{j} \alpha \\
d_{j}
\end{array}\right)
$$

where $\left\{x_{j}\right\} \subseteq\left\{a_{i}\right\}$ (possible since $|J| \leqslant|I|$ ). Then $\beta=\lambda \alpha \mu$ and $\lambda, \mu \in R(q)$ (note that if $q<p$ then we can assume $I=J$ ). Finally a standard argument shows that if $r(\alpha)=r(\beta)$ then $\alpha \mathcal{D} \beta$, so $\mathcal{J} \subseteq \mathcal{D}$ and equality follows.

REMARK 3. From a comment above, we deduce that $\alpha \mathcal{H} \beta$ in $R(q)$ if and only if $\operatorname{ran} \alpha=\operatorname{ran} \beta$ and $\operatorname{dom} \alpha=\operatorname{dom} \beta$. Hence, as in Remark 1 about $P S(q)$, the group $\mathcal{H}$ classes of $R(q)$ are precisely the symmetric groups $G(A)$ where $A \subseteq X$ and $|X \backslash A|=q$. For the group $\mathcal{H}$-classes of $S_{q}$, note that no idempotent of $P S(q)$ has gap less than $q$, hence Corollary 2 shows that $\mathcal{H}$ in $S_{q}$ can be characterised in the same way as for $R(q)$, and therefore the group $\mathcal{H}$-classes of $S_{q}$ are also the same as for $R(q)$.

\section{TWO-SIDED IDEALS}

Recall that for $q \leqslant r \leqslant p, S_{r}=\{\alpha \in P S(q): g(\alpha) \leqslant r\}$ is a subsemigroup of $P S(q)$. The reverse inequality gives us ideals of $P S(q)$ when $q<p$. 
THEOREM 13. The proper ideals of $P S(q)$ for $q<p$ are precisely the sets:

$$
T_{r}=\{\alpha \in P S(q): g(\alpha) \geqslant r\}
$$

where $q<r \leqslant p$. Moreover, each $T_{r}$ is a principal ideal.

Proof: Let $\alpha \in T_{r}$ and $\beta \in P S(q)$. Since $\operatorname{dom} \alpha \beta \subseteq \operatorname{dom} \alpha$, we know $g(\alpha \beta)$ $\geqslant g(\alpha)$, so each $T_{r}$ is a right ideal. Also,

$$
X \backslash \operatorname{dom} \beta \alpha=(X \backslash \operatorname{dom} \beta) \cup(\operatorname{dom} \beta \backslash \operatorname{dom} \beta \alpha)
$$

and

$$
G(\alpha)=[X \beta \cap G(\alpha)] \cup[(X \backslash X \beta) \cap G(\alpha)]
$$

where $[X \beta \cap G(\alpha)] \beta^{-1}=\operatorname{dom} \beta \backslash \operatorname{dom} \beta \alpha$ and $d(\beta)=q$. Therefore, $|X \beta \cap G(\alpha)| \geqslant r$ and it follows that $g(\beta \alpha) \geqslant r$. That is, $T_{r}$ is also a left ideal.

Conversely, suppose $A$ is a proper ideal of $P S(q)$ for $q<p$ and choose $\alpha \in A$ with least gap, $r$ say, so $A \subseteq T_{r}$. If $r \leqslant q$ then, by Lemma 3, all elements of $P S(q)$ belong to $P S(q) \alpha P S(q)$ which is contained in $A$ : that is, $A=P S(q)$, a contradiction. Therefore $q<r \leqslant p$ and if $\beta \in T_{r}$ then $g(\beta) \geqslant r=g(\alpha)>q$, so Lemma 3 implies $\beta=\lambda \alpha \mu$ for some $\lambda, \mu \in P S(q)$. Hence $\beta \in A$ and equality follows.

Finally, if $\alpha \in T_{r}$ has gap $r$ where $q<r \leqslant p$ then Lemma 3 implies that, for each $\beta \in T_{r}$, there exist $\lambda, \mu \in P S(q)$ such that $\beta=\lambda \alpha \mu$ and hence $T_{r} \subseteq P S(q)^{1} \alpha P S(q)^{1}$. Since $\alpha \in T_{r}$ and $T_{r}$ is an ideal, the reverse inclusion also holds, and thus each $T_{r}$ is principal.

In effect, in [1, Vol. 2, Lemma 10.54], Clifford and Preston prove that the Rees factor semigroups $I_{\xi^{\prime}} / I_{\xi}$ of ideals $I_{\xi}$ in $T(X)$ are 0-bisimple, and they contain a primitive idempotent precisely when $\xi$ is finite (here $\xi^{\prime}$ denotes the successor of the cardinal $\xi)$. To obtain a corresponding result for the ideals of $P S(q)$, we first observe that if $q<r \leqslant s \leqslant p$ then $q^{\prime} \leqslant r$ and

$$
T_{p} \subseteq \cdots \subseteq T_{s} \subseteq T_{r} \subseteq \cdots \subseteq T_{q^{\prime}} .
$$

Note that if $q<r \leqslant p$ then $G_{r}=S_{r} \cap T_{r}$ is the (non-empty) set of all $\alpha \in P S(q)$ with gap $r$, and in fact $G_{r}$ is a semigroup (since it is the intersection of two semigroups). Therefore, if $q<r<p$ then $T_{r} / T_{r^{\prime}}$ is essentially $G_{r}$ with a zero adjoined (note that $\left.G_{p}=T_{p}\right)$.

Remark 4. If $\alpha, \beta$ are $\mathcal{D}$-related in $G_{r}$ then they are $\mathcal{D}$-related in $P S(q)$. Conversely, from Theorem 10 we deduce that if $\alpha, \beta$ are $\mathcal{D}$-related in $P S(q)$ then they have the same gap, $r$ say. Moreover, in this case, $\alpha \mathcal{L} \gamma \mathcal{R} \beta$ for some $\gamma \in P S(q)$ with the 
same gap as $\alpha$ (see the proof of Theorem 10). Now by Theorem 8, either $\alpha=\gamma$ or " $X \alpha=X \gamma$ and $g(\alpha)=g(\gamma) \geqslant q$ "; and in the latter case, as in the second half of the proof of Theorem 8, we can find $\lambda_{1}, \lambda_{2}$ with gap $r$ such that $\alpha=\lambda_{1} \gamma$ and $\gamma=\lambda_{2} \alpha$ : that is, $\alpha \mathcal{L} \gamma$ in $G_{r}$. On the other hand, if $\gamma \mathcal{R} \beta$ in $P S(q)$ then $\operatorname{dom} \gamma=\operatorname{dom} \beta$ by Theorem 7. In addition, if $\gamma$ and $\beta$ have gap $r>q$, we can write

$$
\gamma=\left(\begin{array}{c}
a_{i} \\
b_{i}
\end{array}\right), \quad \beta=\left(\begin{array}{c}
a_{i} \\
c_{i}
\end{array}\right), \quad \mu_{1}=\left(\begin{array}{c}
x_{i} \beta \\
b_{i}
\end{array}\right)
$$

where $\left\{a_{i}\right\}=\left\{x_{i}\right\} \cup \dot{\cup}\left\{x_{k}\right\}$ and $|K|=r$. Then $g\left(\mu_{1}\right)=\left|\left\{x_{k} \beta\right\} \cup X \backslash\left\{c_{i}\right\}\right|=r$ (since $d(\beta)=q<r)$ and $\gamma=\beta \mu_{1}$. That is, if $\gamma \mathcal{R} \beta$ in $P S(q)$ and $q<r=g(\beta) \leqslant p$, we can show that $\gamma \mathcal{R} \beta$ in $G_{r}$. In other words, if $\alpha, \beta$ are $\mathcal{D}$-related in $\operatorname{PS}(q)$ and have gap $r$ where $q<r \leqslant p$ then they are $\mathcal{D}$-related in $G_{r}$.

From the above Remark, we deduce that $G_{r}$ is bisimple if $q<r \leqslant p$. Also, if $\varepsilon$ is an idempotent in $G_{r}$ then $\varepsilon=\operatorname{id}_{A}$ for some $A \subseteq X$ such that $|A|=p$ and $|X \backslash A|=r>q$, which contradicts $d(\varepsilon)=q$. That is, $G_{r}$ is idempotent-free.

COROLLARY 3. If $q<r \leqslant p$ then $G_{r}=S_{r} \cap T_{r}$ is bisimple and idempotent-free.

When $q=p, P S(q)$ contains constant maps, all of which form an ideal of $P S(q)$, so we can expect a more standard description of the ideals in $P S(q)$ in this case: compare [1, Vol. 2, Theorem 10.59] for the ideals of $T(X)$.

THEOREM 14. If $q=p$, the ideals of $P S(q)$ are precisely the sets:

$$
J_{r}=\{\alpha \in P S(q): r(\alpha)<r\}
$$

where $1 \leqslant r \leqslant p^{\prime}$. Moreover, $J_{r}$ is principal precisely when $r=s^{\prime}$ where $0 \leqslant s \leqslant p$.

Proof: Clearly each $J_{r}$ is an ideal of $P S(q)$. Let $A$ be an ideal of $P S(q)$ and let $r$ be the least cardinal greater than $r(\alpha)$ for all $\alpha \in A$. Then $A \subseteq J_{r}$. Now, for each $\beta \in J_{r}$, there exists $\alpha \in A$ such that $r(\beta) \leqslant r(\alpha)$ (by the choice of $r$ ). Hence Lemma 4 implies $\beta=\lambda \alpha \mu$ for some $\lambda, \mu \in P S(q)$, so $\beta \in A$. That is, $J_{r} \subseteq A$ and equality follows. Moreover, if $r=s^{\prime}$ then $J_{r}=\{\alpha \in P S(q): r(\alpha) \leqslant s\}$. In this case, since $p=q$, Lemma 4 implies $J_{r} \subseteq P S(q)^{1} \alpha P S(q)^{1}$ for each $\alpha \in J_{r}$ with rank $s$, and it follows that $J_{r}$ is principal. Conversely, suppose $J_{r}=P S(q)^{1} \alpha P S(q)^{1}$ for some $\alpha \in J_{\mathrm{r}}$. Let $r(\alpha)=s$ and assume there is a cardinal $t$ such that $s<t<r$. Since $p=q$, there exists $\beta \in P S(q)$ with $r(\beta)=t$ and then $\beta \in J_{r}$, so $\beta=\lambda \alpha \mu$ for some $\lambda, \mu \in P S(q)^{1}$. But this implies $r(\beta) \leqslant r(\alpha)$, a contradiction. Therefore, $t$ does not exist and thus $r=s^{\prime}$.

REMARK 5. If non-zero $\alpha, \beta$ are $\mathcal{D}$-related in $J_{r^{\prime}} / J_{r}$ then they are $\mathcal{D}$-related in $P S(q)$. Conversely, from Theorem 10 we deduce that if $\alpha, \beta$ are $\mathcal{D}$-related in $P S(q)$ then they 
have the same rank, $r$ say. Moreover, in this case, $\alpha \mathcal{L} \gamma \mathcal{R} \beta$ for some $\gamma \in P S(q)$ with the same rank $r$ (see the proof of Theorem 10). Next we observe that, in the proof of Theorem 7, $\mu$ has the same rank as $\alpha$, and this can be used to show that, if elements of $P S(q)$ are $\mathcal{R}$-related in $P S(q)$ and have rank $r$, then they are $\mathcal{R}$-related in $J_{r^{\prime}} / J_{r}$. In addition, if $\alpha \mathcal{L} \gamma$ in $P S(q)$ then Theorem 8 implies that either $\alpha=\gamma$ or " $X \alpha=X \gamma$ and $g(\alpha)=g(\gamma) \geqslant q$ "; and in the latter case, as in the second half of the proof of Theorem 8, we can find $\lambda_{1}, \lambda_{2}$ with rank $r$ such that $\alpha=\lambda_{1} \gamma$ and $\gamma=\lambda_{2} \alpha$ : that is, $\alpha \mathcal{L} \gamma$ in $J_{r^{\prime}} / J_{r}$. In other words, if $\alpha, \beta$ are $\mathcal{D}$-related in $P S(q)$ and have rank $r$ then they are $\mathcal{D}$-related in $J_{r^{\prime}} / J_{r}$.

Now, in Example 2 we found $\alpha, \beta$ with rank $p$ which are not $\mathcal{D}$-related in $P S(q)$ and so, by the above Remark, $J_{p^{\prime}} / J_{p}$ is not 0-bisimple. On the other hand, if $r<p=q$ then all non-zero elements of $J_{r^{\prime}} / J_{r}$ have the same rank $r$ and gap $p$, so Theorem 10 implies they are $\mathcal{D}$-related in $P S(q)$ and hence also in $J_{r^{\prime}} / J_{r}$; that is, $J_{r^{\prime}} / J_{r}$ is 0 bisimple if $1 \leqslant r<p$. However, if $\varepsilon$ is a non-zero idempotent in $J_{r^{\prime}} / J_{r}$ then $\varepsilon=\operatorname{id}_{A}$ for some $A \subseteq X$ such that $|A|=r$ and $|X \backslash A|=q$; and, since $A \backslash\{x\} \varsubsetneqq A$ if $x \in A$, this is primitive precisely when $r$ is finite and positive (see [1, Vol. 2, p. 224]). That is, $J_{r^{\prime}} / J_{r}$ is completely 0 -simple only when $1 \leqslant r<\aleph_{0}$. Finally, by Theorem 4 , if each $\alpha$ in $J_{r^{\prime}} / J_{r}$ is regular, we must have $r<q=p$ (since elements with rank $p$ can have gap less than $p$ ). In other words, $J_{r^{\prime}} / J_{r}$ is inverse precisely when $0 \leqslant r<p$.

COROLLARY 4. If $1 \leqslant r<p=q$ then $J_{r^{\prime}} / J_{r}$ is a 0 -bisimple inverse semigroup; it is completely 0 -simple only when $r$ is finite.

Note that if $q<p$ and $\alpha, \beta \in S_{q}$ then $r(\alpha)=r(\beta)=p$, so $\alpha \mathcal{J} \beta$ in $S_{q}$ by Theorem 11(a). Thus, $S_{q}$ is simple if $q<p$, and of course if $q=p$ then $S_{q}=P S(q)$. Likewise if $q<p$ then $R(q)$ is simple (in fact, bisimple since $\mathcal{D}=\mathcal{J}$ when $q<p$ ). And if $q=p$ then $R(q)$ contains constant maps and an argument similar to that in the above proof leads to our last result.

THEOREM 15. If $q=p$, the ideals of $R(q)$ are precisely the sets $R(q) \cap J_{r}$ where $1 \leqslant r \leqslant p^{\prime}$.

\section{REFERENCES}

[1] A.H. Clifford and G.B. Preston, The algebraic theory of semigroups, Mathematical Surveys, No. 7, vol 1 and 2 (American Mathematical Society, Providence, RI, 1961 and 1967).

[2] J.M. Howie, An introduction to semigroup theory (Academic Press, London, 1976).

[3] J.M. Howie, 'A congruence-free inverse semigroup associated with a pair of infinite cardinals', J. Austral. Math. Soc. Ser. A 31 (1981), 337-342.

[4] I. Levi, 'Automorphisms of normal partial transformation semigroups', Glasgow Math. J. 29 (1987), 149-157. 
[5] I. Levi, B.M. Schein, R.P. Sullivan and G.R. Wood, 'Automorphisms of Baer-Levi semigroups', J. London Math. Soc. 28 (1983), 492-495.

[6] D. Lindsey and B. Madison, 'The lattice of congruences on a Baer-Levi semigroup', Semigroup Forum 12 (1976), 63-70.

[7] M.P.O. Marques-Smith and R.P. Sullivan, 'The ideal structure of nilpotent-generated transformation semigroups', Bull. Austral. Math. Soc. 60 (1999), 303-318.

[8] M.A. Reynolds and R.P. Sullivan, 'The ideal structure of idempotent-generated transformation semigroups', Proc Edinburgh Math. Soc. 28 (1985), 319-331.

[9] R.P. Sullivan, 'Automorphisms of transformation semigroups', J. Austral. Math. Soc. 20 (1975), 77-84.

[10] R.P. Sullivan, 'Semigroups generated by nilpotent transformations', J. Algebra 110 (1987), 324-343.

Centro de Matematica

Universidade do Minho

4710 Braga

Portugal
School of Mathematics \& Statistics

University of Western Australia

Nedlands W.A. 6009

Australia 